\title{
Plant community analysis along environmental gradients in moist afromontane forest of Gerba Dima, South-western Ethiopia
}

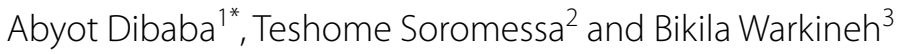

\begin{abstract}
Background: This study was carried out in Gerba Dima Forest, South-Western Ethiopia, to determine the floristic composition, species diversity and community types along environmental gradients. Identifying and interpreting the structure of species assemblages is the main goal of plant community ecology. Investigation of forest community composition and structure is very useful in understanding the status of tree population, regeneration, and diversity for conservation purposes.

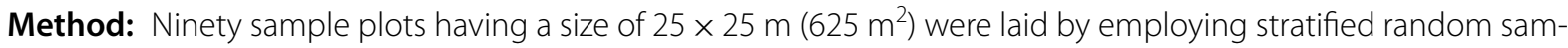
pling. Nested plots were used to sample plants of different sizes and different environmental variables. All woody plant species with Diameter at breast height $(\mathrm{DBH}) \geq 2.5 \mathrm{~cm}$ and height $\geq 1.5 \mathrm{~m}$ were recorded in $25 \mathrm{~m} \times 25 \mathrm{~m}$ plots. Hierarchical (agglomerative) cluster analysis was performed using the free statistical software R version 3.6.1 using package cluster to classify the vegetation into plant community types. Redundancy Analysis (RDA) ordination was used in describing the pattern of plant communities along an environmental gradient.
\end{abstract}

Result: One hundred and eighty plant species belonging to 145 genera, 69 families and comprising of 15 endemic species were recorded. Of these, 52 species (28.9\%) were trees, 6 species (3.33\%) were Trees/shrubs, 31 species (17.22\%) were shrubs, 76 species (42.22\%) were herbs, and 15 species (8.33\%) were Lianas. Rubiaceae, Acanthaceae and Asteraceae were the richest family each represented by 11 genera and 11 species (6.11\%), 9 genera and 11 species (6.11\%), 6 genera and 11 species (6.11\%), respectively of total floristic composition. Cluster analysis resulted in five different plant communities and this result was supported by the ordination result. RDA result showed altitude was the main environmental variable in determining the plant communities. The ANOVA test indicated that the five community types differ significantly from each other with regard to Electrical Conductivity and Potassium.

Conclusions: Description of floristic diversity of species in Gerba Dima forest revealed the presence of high species diversity and richness. The presence of endemic plant species in the study forest shows the potential of the area for biodiversity conservation.

Keywords: Gerba Dima, Indicator species, Moist afromontane forest, Species diversity

${ }^{*}$ Correspondence: abyotdibaba77@yahoo.com

${ }^{1}$ College of Natural Sciences, Department of Biology, Debre Berhan

University, P. O. Box 445, Debre Berhan, Ethiopia

Full list of author information is available at the end of the article

\section{Background}

Identifying and interpreting the structure of species assemblages is the main goal of plant community ecology. Gradients in species composition vis-à-vis either 
presence/absence or abundance data are commonly employed to evaluate community structure [1]. Legendre [2] distinguished between 'true gradients' in species composition, which are induced by environmental gradients, and 'false gradients', which may arise even in the absence of environmental heterogeneity as a result of biotic interactions within the community. Both true and false gradients may form distinct spatial patterns when mapped into geographic space. According to Seabloom et al. [3], different ecological processes create distinct spatial patterns, so that specific processes could be identified from their spatial signature. Hence, spatial analysis of community structure is of direct scientific interest, because spatial structures may be critical for identifying and understanding the underlying ecological processes [4]. Biotic filters determining limiting similarity is the assumed cause for species dissimilarity in traits within communities. Symmetric competitive interactions might indeed lead to the co-existence of ecologically distinct species, which minimize competition for shared resources ("symmetric competition" leading to limiting similarity [5].

The quest to explain the underlying processes for the assembly of local communities is still a major focus in plant community ecology, as researchers keep examining them through both observational and experimental studies [6]. The multidimensional ecological niche space determines the distribution of a species within a community [7]. Physiographic and edaphic factors can determine which plant species will colonize a site since plant species vary in their tolerance and utilization of resources site [8]. These variations have been regarded as a driving force for the coexistence of species in a similar environment [9] and can explain broad-scale compositional differences among multiple resource gradients $[10,11]$. The upper storey tree density as an abiotic factor can also affect community composition as understorey species differ in their ability to tolerate stresses imposed by competitive trees $[12,13]$. Moreover, by increasing the abundance of annual and biennial plants, disturbances can affect community composition via favouring stress-tolerant species $[13,14]$.

Information on species composition and diversity of tree species plays a pivotal role not only to understand the structure of a forest community but also in planning and implementation of conservation strategy of the community [15]. Investigation of forest community composition and structure is very useful in understanding the status of tree population, regeneration, and diversity for conservation purposes [16]. Quantitative information on composition, distribution, and abundance of woody species has paramount importance in understanding the form and structure of a forest community and for planning and implementation of conservation strategy of the community.

The recent data on forest resources of Ethiopia reported in FAO [17] puts Ethiopia among countries with a forest cover of $10-30 \%$. According to this report, Ethiopia's forest cover (FAO definition) is 12.2 million ha (11\%). It further indicated that the forest cover shows a decline from 15.11 million ha in 1990 to 12.2 million ha in 2010, during which $2.65 \%$ of the forest cover was deforested. This study was conducted in the Gerba Dima forest found in South-Western Ethiopia with the aim of investigating the species composition, species diversity, community types and to relate the distribution of plant community types to some environmental parameters.

\section{Methods}

\section{The study area}

This study was carried out in the Gerba Dima forest found in the Illu Aba Bora zone of Oromia regional state of Ethiopia and located between $7^{\circ} 45^{\prime}$ to $8^{\circ} 10^{\prime}$ North latitude and $35^{\circ} 29^{\prime}$ to $35^{\circ} 50^{\prime}$ East longitude. The study forest is bounded by Baro River to the south and west direction whiles three other rivers, namely Bote, Hoyi and Sor cross part of the forest in the east (Fig. 1). The geology of the study site is characterized by the Underlying basement rock consisting of intensively folded and faulted Precambrian rocks, overlain by Mesozoic marine strata and Tertiary basalt types [18]. The main soil types of the study area are red or brownish ferrisols derived from the volcanic parent material. Other soil groups in the area include nitosols, acrisols, vertisols, and cambisols soil types exist in the study site [19].

The rainfall data collected from the nearest Gore meteorological station to the study forest indicated that the study area receives very high annual rainfall and characterized by unimodal rainfall pattern, which shows low rainfall in December, January and February, gradually increasing to the peak period in August. The mean annual rainfall of $1854 \mathrm{~mm}$ while the monthly mean maximum and mean minimum temperature of the area is $27.2^{\circ} \mathrm{C}$ and $13.3{ }^{\circ} \mathrm{C}$, respectively. The mean annual temperature is $19.2{ }^{\circ} \mathrm{C}$ and with slight variation from year to year [20].

The vegetation type at Gerba Dima is part of the moist evergreen afromontane forest with characteristic emergent species that form the upper canopy includes Pouteria adolfi-friederici (Fig. 2). Albizia gummifera, A. schimperiana, A. grandibracteata, Sapium ellipticum, Euphorbia ampliphylla, Ekebergia capensis, Ficus sur, Hallea rubrostipulata, Ocotea kenyensis, Olea welwitschii, Polyscias fulva and Schefflera abyssinica are other characteristic species of this vegetation type [21]. 


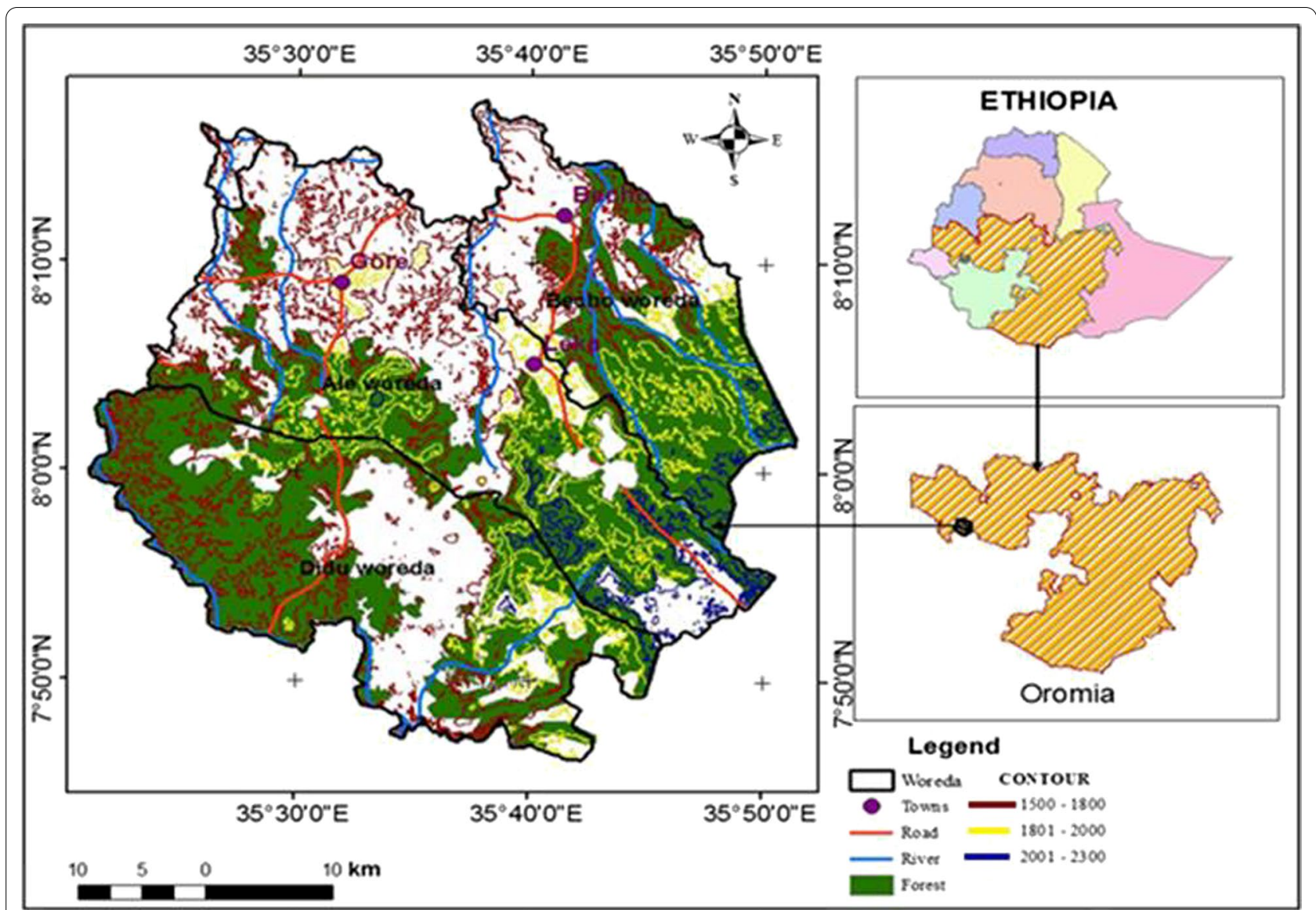

Fig. 1 Map of Ethiopia, Oromia region and Gerba Dima forest
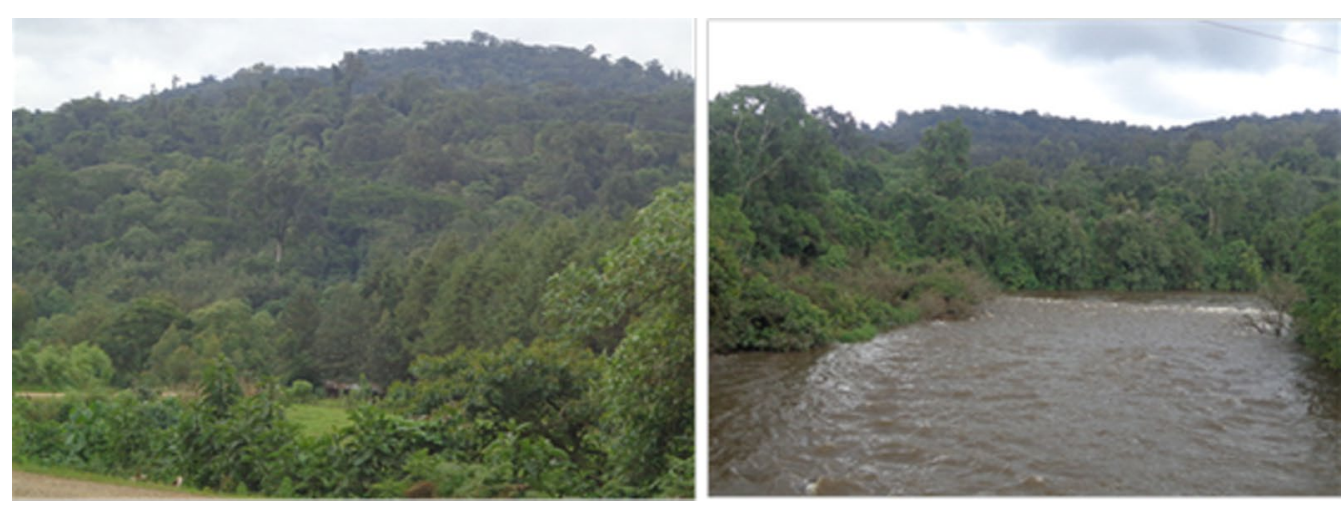

Fig. 2 Photograph illustrating the forest overview

\section{Sampling method}

In this study, a stratified random sampling design was used to collect vegetation and environmental data $[1,22]$. Using Arc GIS version 10.3, the study forest was stratified based on the altitudinal gradient and three types of strata in the form of contour were established. Strata one was distributed between 1500 and $1800 \mathrm{~m}$ altitudinal ranges whereas strata two and three were found between 1801$2000 \mathrm{~m}$ and 2001-2300 $\mathrm{m}$ altitudinal ranges respectively (Fig. 1). Sample plots were assigned in each contour in the form of Random points Using Arc GIS version 10.3 (Fig. 3). 


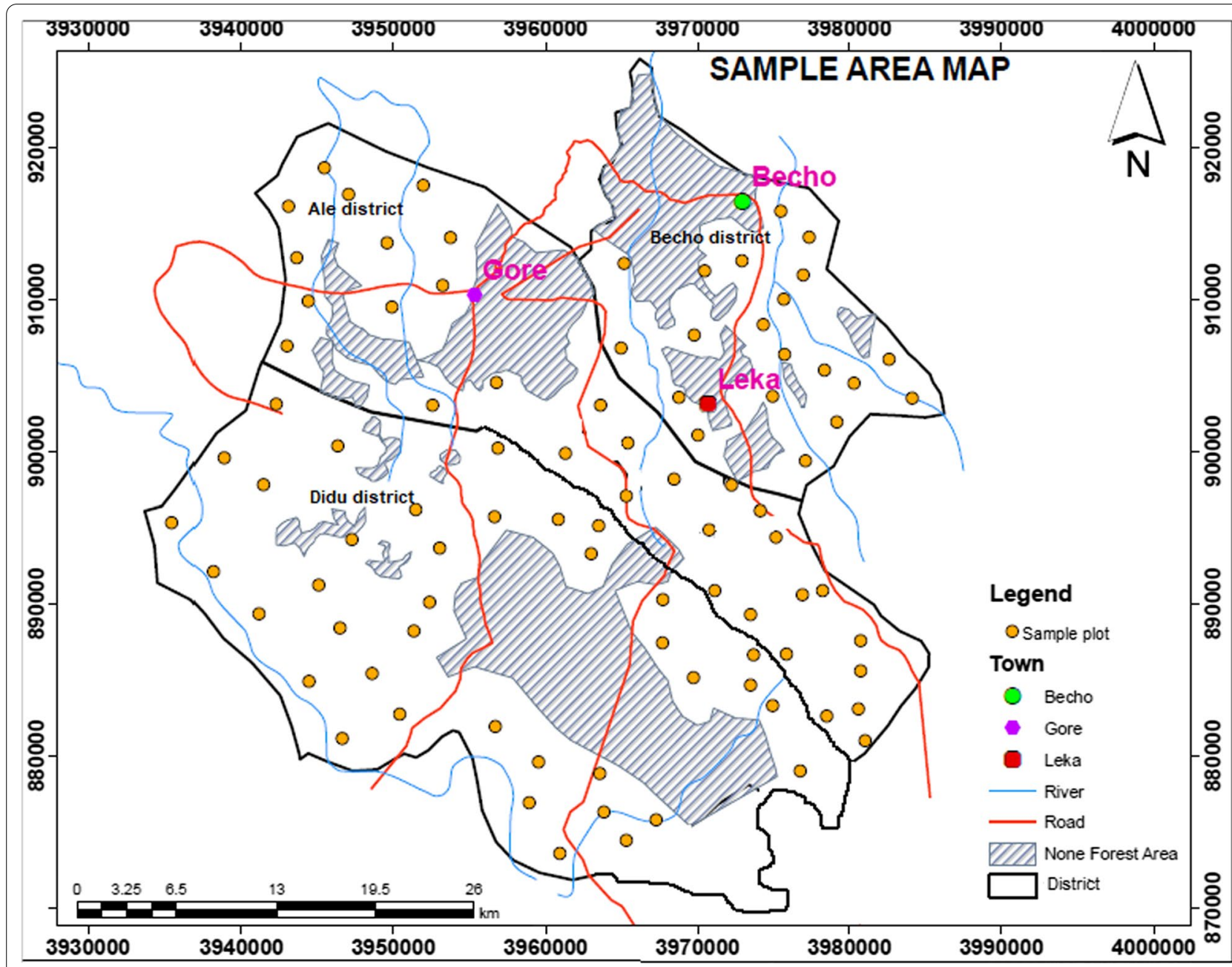

Fig. 3 A Map showing the distribution of sample plots

Ninety sample plots having a size of $25 \times 25 \mathrm{~m}$ $\left(625 \mathrm{~m}^{2}\right)$ along each contour were laid. Nested plots were used to sample plants of different sizes and different environmental variables. All woody plant species with Diameter at breast height $(\mathrm{DBH}) \geq 2.5 \mathrm{~cm}$ and height $\geq 1.5 \mathrm{~m}$ were recorded in $25 \mathrm{~m} \times 25 \mathrm{~m}$ plots. Within the major plots, five $3 \mathrm{~m} \times 3 \mathrm{~m}$ subplots $\left(9 \mathrm{~m}^{2}\right)$ was used to collect shrubs with $\mathrm{dbh}<2.5 \mathrm{~cm}$ and $>1.5 \mathrm{~m}$ height. Within each $9 \mathrm{~m}^{2}$ subplots, two $1 \mathrm{~m}^{2}$ subplots were used to collect data on the species and abundance of herbaceous plants. Finally, the percent cover of all plant species found within the sample plot was visually estimated and converted to the Braun-Blanquet scale as modified by [23]. Every plant species encountered in each plot were recorded. Plant specimens were collected, pressed, dried and brought to the National Herbarium (ETH), Addis Ababa University for taxonomic identification. The specimens were determined by comparing with authenticated specimens housed at ETH and by referring to published volumes of Flora of Ethiopia and Eritrea [24-32].

Physiographical variables, namely altitude, geographic coordinates, slope and aspect, were recorded for each quadrat using GPS, Clinometer and Compass respectively. The values for aspect were codified based on Woldu [33], where $\mathrm{N}=0, \mathrm{NE}=1, \mathrm{E}=2, \mathrm{SE}=3$, $\mathrm{S}=4, \mathrm{SW}=3.25, \mathrm{~W}=2.5, \mathrm{NW}=1.25$ before analysis. For each sample plot, a disturbance was determined on the basis of a five point scale following [34]. The five scales of disturbance scores were based on visible signs of tree cutting, grazing and presence of beehives. The points of scale were $0=$ (No disturbance), $1=(0-20 \%$ of the quadrat disturbed), $2=(21-40 \%$ of the quadrat disturbed), $3=(41-60 \%$ of the quadrat disturbed), $4=(61-80 \%$ of the quadrat disturbed $), 5=(81-100 \%$ of the quadrat disturbed). 
For analysing soil variables, soil samples were collected with a soil core sampler from the top $40 \mathrm{~cm}$ depth within $1 \mathrm{~m} \times 1 \mathrm{~m}$ subplots at the four corners and middle of the quadrat. Composite soil samples from samples collected from the four corners and the middle of quadrats were brought to the soil laboratories of Addis Ababa University (AAU). The soil samples were air-dried, rolled and passed through a $2 \mathrm{~mm}$ sieve for laboratory analyses. These soil samples were analysed for $\mathrm{pH}$, electrical conductivity (EC), sodium, potassium, organic matter, total nitrogen, available phosphorus and texture following standard procedures outlined in [35]. The $\mathrm{pH}$ and $\mathrm{EC}$ were measured using a $\mathrm{pH}$ meter and EC meter in the supernatant suspension of 1:2.5 soil-distilled water mixtures. Available Sodium and Potassium were determined using a flame photometer. Organic matter was determined by the ignition method. The texture was determined on the basis of Bouycous Hydrometer method with the categories sand, silt, and clay (expressed as \% weight) while total nitrogen was determined using Kjeldhal method. Available Phosphorus was determined by the Bray-I method and the absorbance of the Bray-I extract is measured at $882 \mathrm{~nm}$ in a spectrophotometer.

\section{Data analysis}

In this study, hierarchical (agglomerative) cluster analysis was performed using the free statistical software $\mathrm{R}$ version 3.6.1 [36] using package cluster to classify the vegetation into plant community types. The similarity ratio with Ward's group linkage method was applied for cluster analysis i.e. to determine plots that can be classified into the same groups based on the species abundance data. The decision on the number of groups (clusters) was based on objective methods of obtaining an optimal number of clusters, the Multi Response Permutation Procedures (MRPP) technique (no-difference hypothesis) and the ecological interpretation of the groups conducted in $\mathrm{R}$ program. The $\mathrm{T}$ and A statistic of MRPP output were used to obtain the number of clusters. The test statistic $\mathrm{T}$ describes the separation between the groups. The more negative $\mathrm{T}$ value, the stronger the separation. The P-value associated with $\mathrm{T}$ is determined by numerical integration of the Pearson type III distribution. The P-value is useful for evaluating how likely an observed difference is due to chance [37]. The agreement statistic A describes within-group homogeneity, compared to the random expectation, and falls between 0 and 1 . When all items within-groups are identical $\mathrm{A}=1$ and 0 if the groups are heterogeneous. In community ecology, A values are commonly below 0.1 , and an A value greater 0.3 is fairly high [37].

From the output of the objective method, a sharp bend at the specific cluster in the plot could be a good indication of the number of clusters in the data [38]. The community types identified from the cluster analysis were further refined in a synoptic table where species occurrences were summarized as synoptic cover-abundance values [39]. Dominant species of each community type were identified based on their synoptic values and community types were named after one or more dominant species. The identified groups were tested for the hypothesis of no difference between the groups (clusters) using nonparametric Multi-Response Permutation Procedure (MRPP). Indicator species analysis was performed in $\mathrm{R}$ using package labdsv. Indicator values were tested for statistical significance using a randomization (Monte Carlo) technique. Species richness, evenness, Shannon diversity and evenness indices were computed using the free statistical software R version 3.6.1 [36]. The Shannon diversity index $\left(\mathrm{H}^{\prime}\right)$ was calculated from the equation:

$$
H^{\prime}=-\sum_{i=1}^{s} p i \operatorname{lnp} i
$$

where pi, is the proportion of individuals found in the ith species. The values of the Shannon diversity index is usually found to fall between 1.5 and 3.5 and only rarely surpasses $4.5[1,39]$. The Shannon evenness index (J) was calculated from the ratio of observed diversity to maximum diversity using the equation:

$$
J=\frac{H^{\prime}}{H \max }=\frac{H^{\prime}}{\ln s}
$$

where Hmax is the maximum level of diversity possible within a given population, which equals ln (number of species). $J$ is normal between 0 and 1 , and with 1 representing a situation in which all species are equally abundant [40].

Information about endemic species, their habit, IUCN status and geographical distributions was determined by referring to [25-33, 42].

In this study, Redundancy Analysis (RDA) ordination was used in describing the pattern of plant communities along an environmental gradient since the preliminary analysis of the vegetation data using Deterended Correspondence Analysis (DCA) revealed that the longest axis of DCA for the dataset was less than $3(=2.22)$. Before the application of RDA ordination, environmental variables, which were relatively more important in explaining the species data, were selected using the Monte Carlo technique and function Adonis test for their significance. Computation of variance inflation factor (vif) was also conducted to eliminate those environmental variables that are collinear. The community types obtained were subjected to an ANOVA based on environmental variables to find out whether there are significant variations 
between the groups. Pearson's product-moment correlation coefficient was calculated to evaluate the relationship between the environmental variables.

\section{Results}

\section{Floristic composition}

One hundred and eighty (180) plant species belonging to 145 genera and 69 families were recorded and identified in the sample plots in the Gerba Dima forest (Table 1). Of these, 52 species (28.9\%) were trees, 6 species (3.33\%) were Trees/shrubs, 31 species $(17.22 \%)$ were shrubs, 76 species $(42.22 \%)$ were herbs, and 15 species $(8.33 \%)$ were Lianas. Angiosperms were represented by 160 species while the rest 20 species were Pteridophytes. Among Angiosperms, Rubiaceae, Acanthaceae and Asteraceae were the richest family each represented by 11 genera and 11 species $(6.11 \%), 9$ genera and 11 species $(6.11 \%)$, 6 genera and 11 species $(6.11 \%)$, respectively of total floristic composition, followed by Fabaceae 8 genera and 9 species (5\%), Euphorbiaceae 6 genera and 7 species (3.89\%). The remaining families represented less than 3\% of species each. Eleven families, 13 genera and 20 species represented pteridophytes. Aspleniaceae, Dryopteridaceae and Pteridaceae were the richest Pteridophytes represented by 6,3 and 2 species respectively. The genus Vernonia, Ficus, Asparagus, Dracaena were represented by 5,4,3,3 species respectively and Aframomum, Albizia, Asparagus, Cyperus, Euphorbia, Hippocratea, Hypoestes, Justicia, Maytenus, Olea, Peperomia, Polyscias, Pteris, Rubus, Schefflera, Solanecio, Solanum, Tacazzea, and Zehneria were represented by 2 species each and the rest genera contained a single species each.

Based on the information available on the published Floras of Ethiopia a total of 15 endemic plant species in 11 families were recorded (Table 2), comprising more than $8.33 \%$ of the recorded species. Asteraceae was the first family having three endemic species, followed by Acanthaceae and Fabaceae (two species each). The remaining eight families have a single species each in the endemic species list. Among the total endemic species, herb, tree, shrub and liana growth forms were represented by 6,3,4,2 species respectively. Out of the 15 endemic species, Crotalaria rosenii and Polyscias farinosa have been included in the IUCN red data list of Ethiopia and Eritrea qualifying for near threatened and vulnerable category respectively. In the Gerba Dima forest, at $625 \mathrm{~m}^{2}$ sample plot, species richness varied from 26 to 59 across the study plots. The Shannon diversity index also varied from 2.92 to 3.83 while evenness ranged from 0.89 to 0.95 in the study plots. The overall mean Shannon diversity index, species richness and evenness of the study area were 3.45, 41 and 0.93 respectively.

\section{Community types and indicator species}

Five community types were derived from the hierarchical cluster analysis in combination with Multi-response Permutation Procedures (MRPP) and objective method of the whole data set (Fig. 4 and Table 3). From the output of MRPP, the test statistic $\mathrm{T}$ value for the five groups was $-38.26(\mathrm{P}<0.001)$ and the agreement statistic $\mathrm{A}$ was 0.13 while the output of objective method revealed a sharp bend at the fifth cluster.

Community 1 (Croton macrostachyus-Bersama abyssinica community) was found in the altitudinal range of $1677-2020$ m. a.s.l and slope from flat to 50\%. Fourteen plots were associated with the community and has 2 indicator species with significant indicator values $(\mathrm{P}<0.05)$ (Table 4).

Community 2 (Syzygium guineense-Olea capensis community) was distributed from 1699 to $2240 \mathrm{~m}$ a.s.l. and slope ranging from flat to $60 \%$. It comprises of 22 plots and twenty species were associated with this community as indicator species where one of the indicator species exhibit significant indicator values $(\mathrm{P}<0.05)$ (Table 4).

Community 3 (Dracaena afromontana- Pouteria adolfi-friederici community) was found in the altitudinal range of $1761-2000 \mathrm{~m}$. a.s.l and slope from flat to $25 \%$. Thirteen plots were associated with the community community and seven species were associated with this community as indicator species while two of the indicator species showed significant indicator values $(\mathrm{P}<0.05)$ (Table 4).

Community 4 (Vepris dainellii-Schefflera abyssinica community) was distributed in the altitude range of $1720-2060 \mathrm{~m}$ a.s.l. and the slope gradient varies flat to $60 \%$. It comprised of 14 plots, eight species were associated with this community as indicator species, while four of the indicator species exhibited significant indicator values $(\mathrm{P}<0.05)$ (Table 4$)$.

Community 5 (Albizia gummifera-Millettia ferruginea community) was found in the altitudinal range of 1728 $2014 \mathrm{~m}$. a.s.l and slope from flat to $50 \%$. Twenty-seven plots were associated to the community. Eight species are associated with this community as indicator species and four of the indicator species exhibited significant indicator values $(\mathrm{P}<0.05)$ (Table 4$)$.

From computation of vegetation data in the study area Shannon-Weiner diversity and evenness, indices for the five community types showed the output in Table 5 .

\section{Relationship between community types and environmental factors}

Heterogeneity or homogeneity of vegetation data test using DCA resulted in short length (gradient) of DCA 
Table 1 List of species in Gerba Dima Forest

\begin{tabular}{|c|c|c|c|c|c|}
\hline No & Scientific names & Family & Local names $^{\mathrm{a}}$ & Habit & Voucher No. \\
\hline 1 & Acanthopale ethio-germanica Ensermu & Acanthaceae & Dargu & S & AD005 \\
\hline 2 & Acanthus eminens C.B.Clarke & Acanthaceae & Qosambe booyyee & S & AD107 \\
\hline 3 & Achyranthes aspera $\mathrm{L}$ & Amaranthaceae & Maxxane & $\mathrm{H}$ & AD160 \\
\hline 4 & Achyrospermum schimperi (Hochst. ex Briq.) Perkins & Lamiaceae & - & $\mathrm{H}$ & AD134 \\
\hline 5 & Adiantum poiretii Wikstr & Adiantaceae & & $\mathrm{H}$ & AD120 \\
\hline 6 & Aerangis brachycarpa (A. Rich) Th Dur.\& Schinz & Orchidaceae & - & $\mathrm{H}$ & AD062 \\
\hline 7 & Aframomum corrorima (Braun) Jansen & Zingiberaceae & Ogiiyo & $\mathrm{H}$ & AD045 \\
\hline 8 & Aframomum zambesiacum (Baker) K. Schum & Zingiberaceae & Ogiiyo jaldessaa & $\mathrm{H}$ & AD096 \\
\hline 9 & Ageratum conyzoides $L$ & Asteraceae & - & $\mathrm{H}$ & AD038 \\
\hline 10 & Ajuga sp. (=Friis et al. 1456) & Lamiaceae & Gondii & $\mathrm{H}$ & AD118 \\
\hline 11 & Alangium chinense (Lour.) Harms & Alengeaceae & Hudu fardaa/sendo & $\mathrm{T}$ & AD007 \\
\hline 12 & Albizia gummifera (J.F. Gmel.) C.A. Sm., & Fabaceae & Ambabbessa dhaltu & $\mathrm{T}$ & AD078 \\
\hline 13 & Albizia schimperiana Oliv & Fabaceae & Ambabbessa kormaa & $\mathrm{T}$ & AD009 \\
\hline 14 & Alchemilla abyssinica Fresen & Roseaceae & Korbesso & $\mathrm{H}$ & AD013 \\
\hline 15 & Allophyllus abyssinicus (Hochst.) Radlk & Sapindaceae & Se'o & $\mathrm{T}$ & AD021 \\
\hline 16 & Antrophyum mannianum Hook & Vittariaceae & Gixoo & $\mathrm{H}$ & AD082 \\
\hline 17 & Apodytes dimidiata E. Mey. ex Arn & Icaccinaceae & Wandabiyo & $\mathrm{T}$ & AD123 \\
\hline 18 & Arisaema mooneyanum Gilbert \& Mayo & Araceae & Kiicu & $\mathrm{H}$ & AD144 \\
\hline 19 & Asparagus africanus Lam & Asparagaceae & Sariiti & $\mathrm{H}$ & AD165 \\
\hline 20 & Asparagus flagellaris (Kunth) Baker & Asparagaceae & Sariiti & $\mathrm{H}$ & AD180 \\
\hline 21 & Asparagus setaceus (Kunth) Jessop & Asparagaceae & Sariiti & $\mathrm{H}$ & AD174 \\
\hline 22 & Asplenium aethiopicum (Burm.f.) Bech & Aspleniaceae & - & $\mathrm{H}$ & AD179 \\
\hline 24 & Asplenium bugoiense Hieron & Aspleniaceae & Giixoo & $\mathrm{H}$ & AD143 \\
\hline 23 & Asplenium elllottii C.H.Wright, & Aspleniaceae & Giixoo & $\mathrm{H}$ & AD122 \\
\hline 25 & Asplenium erectum Bory ex Willd & Aspleniaceae & - & $\mathrm{H}$ & AD101 \\
\hline 27 & Asplenium sandersonii Hook & Aspleniaceae & Giixoo & $\mathrm{H}$ & AD083 \\
\hline 26 & Asplenium warnetkei Hieron & Aspleniaceae & Giixoo & $\mathrm{H}$ & AD042 \\
\hline 28 & Bersama abyssinica Fresen & Melianthaceae & Lolchisaa & T & AD024 \\
\hline 29 & Bothriocline schimperi Oliv. \& Hiern exBenth & Asteraceae & Ilbu & S & AD129 \\
\hline 30 & Brillantaisia madagascariensis T. Anders. ex Lindau & Acanthaceae & Huxii & S & AD037 \\
\hline 31 & Brucea antidysenterica J. F. Mill & Simaroubaceae & Qomanyo & $\mathrm{T}$ & AD041 \\
\hline 32 & Canthium oligocarpum Hiern & Rubiaceae & Mixo & $\mathrm{S}$ & AD029 \\
\hline 33 & Cassipourea malosana (Baker) Alston & Rhizophoraceae & Looko & $\mathrm{T}$ & AD046 \\
\hline 34 & Cayratia gracilis (Guill. \& Perr.) Suesseng & Vitaceae & Kalaalaa qamale & $\mathrm{H}$ & AD093 \\
\hline 35 & Celtis africana Burm.f & Ulmaceae & Ceeyii & $\mathrm{T}$ & AD015 \\
\hline 36 & Chionanthus mildbraedii (Gilg \& Schellenb.) Stearn & Oleaceae & Kara waayyu & $\mathrm{T}$ & AD004 \\
\hline 37 & Cissampelos mucronata A.Rich & Menispermaceae & - & $\mathrm{L}$ & AD008 \\
\hline 38 & Clausena anisata (Wild.) Benth & Rutaceae & Ulmaayye & $\mathrm{S}$ & AD087 \\
\hline 39 & Clerodendrum myricoides (Hochst.) Varlee, & Lamiaceae & Maraasisaa & $\mathrm{S}$ & AD099 \\
\hline 40 & Clematis longicauda Steud. ex A. Rich & Ranuaculaceae & Fiitii & $\mathrm{L}$ & AD002 \\
\hline 42 & Coffea arabica L & Rubiaceae & Buna & $\mathrm{T} / \mathrm{S}$ & AD010 \\
\hline 41 & Coleochloa abyssinica (Hochsl. ex A Rick) Gilly & Cyperaceae & Coqorsa mukaa & $\mathrm{H}$ & AD019 \\
\hline 43 & Combretum paniculatum Vent & Combreataceae & Bagge & $L$ & AD177 \\
\hline 44 & Commelina diffusa Burm.f & Commelinaceae & Qorxabo & $\mathrm{H}$ & AD161 \\
\hline 45 & Coniogramme africana Heiron & Hemionitidaceae & - & $\mathrm{H}$ & AD006 \\
\hline 46 & Cordia africana Lam & Boraginaceae & Waddessa & $\mathrm{T}$ & AD110 \\
\hline 47 & Crotalaria rosenii (Pax) Milne-Redh. ex Polhill & Fabaceae & Ceekaa & $\mathrm{H}$ & AD147 \\
\hline 48 & Croton macrostachyus Del & Euphorbiaceae & Makkanisa & T & AD030 \\
\hline 49 & Cucumis dipsaceus Ehrenb. ex Spach & Cucurbitaceae & Umbaa'oo & $\mathrm{H}$ & AD036 \\
\hline
\end{tabular}


Table 1 (continued)

\begin{tabular}{|c|c|c|c|c|c|}
\hline No & Scientific names & Family & Local names $^{\mathrm{a}}$ & Habit & Voucher No. \\
\hline 50 & Culcasia falcifolia Engl & Araceae & Qasso & $\mathrm{H}$ & AD077 \\
\hline 51 & Cyathea manniana Hook & Cyatheaceae & Sesino & $\mathrm{T}$ & AD074 \\
\hline 52 & Cyperus fischerianus A. Rich & Cyperaceae & Qunni & $\mathrm{H}$ & AD126 \\
\hline 53 & Cyperus longus L & Cyperaceae & - & $\mathrm{H}$ & AD011 \\
\hline 54 & Dalbergia lactea Vatke & Fabaceae & Sarxe dhittaa & $\mathrm{S}$ & AD018 \\
\hline 55 & Deinbollia kilimandscharica Taub & Sapindaceae & Qaso & $\mathrm{T}$ & AD017 \\
\hline 56 & Desmodium repandum (Vahl)DC & Fabaceae & Maxxanne & $\mathrm{H}$ & AD033 \\
\hline 57 & Didymochlaena truncatula (Sw.)J.Sm & Dryopteridaceae & - & $\mathrm{H}$ & AD035 \\
\hline 58 & Dombeya torrida (J.F. Gmel.) P.Bamps & Sterculiaceae & Daanisaa & $\mathrm{S}$ & AD034 \\
\hline 63 & $\begin{array}{l}\text { Doryopteris concolor (Langsd \& Fisch.) Kuhn } \\
\text { in von der Deck.efl }\end{array}$ & Dryopteridaceae & - & $\mathrm{H}$ & AD051 \\
\hline 59 & Dracaena afromontana Mildbr & Dracenaceae & Sarxe & $\mathrm{T} / \mathrm{S}$ & AD072 \\
\hline 60 & Dracaena fragrans (L.) Ker Gawl & Dracenaceae & Sarxe & S & AD090 \\
\hline 61 & Dracaena steudneri Engl & Dracenaceae & Sarxe & $\mathrm{T}$ & AD108 \\
\hline 62 & Drynaria volkensii Hieron & Polypodiaceae & Balessa & $\mathrm{H}$ & AD012 \\
\hline 64 & Ehretia cymosa Thonn & Boraginaceae & Ulaagaa & $\mathrm{T}$ & AD026 \\
\hline 65 & Ekebergia capensis Sparrm & Meliaceae & Sombo & $\mathrm{T}$ & AD061 \\
\hline 66 & Elaeodendron buchananii (Loes.) Loes & Celastraceae & Waaso & $\mathrm{T}$ & AD167 \\
\hline 67 & Elastostema monticolum Hook.f & Urticaceae & - & $\mathrm{H}$ & AD162 \\
\hline 68 & Ensete ventericosum (Welw.) Cheesman & Musaceae & Eeppoo & $\mathrm{H}$ & AD171 \\
\hline 69 & Erythrococca trichogyne (Muell. Arg.) Prain & Euphorbiaceae & Caakkoo & $\mathrm{T} / \mathrm{S}$ & AD032 \\
\hline 70 & Euphorbia ampliphylla Pax & Euphorbiaceae & Adaami & $\mathrm{T}$ & AD040 \\
\hline 71 & Euphorbia schimperiana Scheele & Euphorbiaceae & Ananno & $\mathrm{S}$ & AD064 \\
\hline 72 & Ficus exasperata Vahl & Moraceae & Baalaantaayii & $\mathrm{T}$ & AD068 \\
\hline 73 & Ficus ovata Vahl & Moraceae & Qilxu & $\mathrm{T}$ & AD065 \\
\hline 74 & Ficus sur Forssk & Moraceae & Harbu & $\mathrm{T}$ & AD073 \\
\hline 75 & Ficus thonningii Blume & Moraceae & Dambii & $\mathrm{T}$ & AD136 \\
\hline 76 & Flacourtia indica (Burm.f.) Merr & Flacourtiaceae & Akuku & $\mathrm{T}$ & AD139 \\
\hline 77 & Galiniera saxifraga (Hochst.) Bridson & Rubiaceae & Simararu & $\mathrm{T}$ & AD137 \\
\hline 78 & Glycine wightii (Wight '\& Am) Verde & Fabaceae & Kalaalaa & $\mathrm{H}$ & AD170 \\
\hline 79 & Gouania longispicata Engl & Rhaminaceae & Hidda reffaa & $L$ & AD020 \\
\hline 80 & Hallea rubrostipulata (K. Schum.) J.-F. Leroy & Rubiaceae & Oobo/Bootto & T & AD016 \\
\hline 81 & Hibiscus panduriformis Burm.f & Malviaceae & Dabbasee & $\mathrm{H}$ & AD163 \\
\hline 82 & Hippocratea africana (Willd.) Loes & Celastraceae & Xiyo & $L$ & AD166 \\
\hline 83 & Hippocratea pallens Planch ex Oliver & Celastraceae & Qawo & $\mathrm{L}$ & AD121 \\
\hline 84 & Hypoestes forskaolii (Vahl) R. Br & Acanthaceae & Dargu & $\mathrm{H}$ & AD124 \\
\hline 85 & Hypoestes triflora (Forssk.) Roem \& Schult & Acanthaceae & Dargu & $\mathrm{H}$ & AD135 \\
\hline 86 & Ilex mitis (L.) Radlk & Aquifoliaceae & Qato & $\mathrm{T}$ & AD155 \\
\hline 87 & Ipomea indica (Burm. f) Merrill & Convolvulaceae & Kalaalaa & $\mathrm{H}$ & AD148 \\
\hline 88 & Isoglossa somalensis Lindau & Acanthaceae & Illbu & $\mathrm{H}$ & AD001 \\
\hline 89 & Jasminum abyssinicum Hochst. ex DC & Oleaceae & Ilchime & $L$ & AD080 \\
\hline 90 & Justicia bizuneshiae Ensermu & Acanthaceae & - & $\mathrm{H}$ & AD059 \\
\hline 91 & Justicia schimperiana (Hochst. ex Nees) T. Anders & Acanthaceae & Dhumugaa & $\mathrm{S}$ & AD053 \\
\hline 92 & Kalanchoe petitiana A. Rich & Crassulaceae & Bosoqe mukaa & $\mathrm{H}$ & AD044 \\
\hline 93 & Keetia gueinzii (Sond.) Bridson & Rubiaceae & Halale & $\mathrm{T} / \mathrm{S}$ & AD111 \\
\hline 94 & Lagera crispata (Vahl) Hepper \& Wood & Asteracea & - & $\mathrm{H}$ & AD117 \\
\hline 95 & Landolphia buchananii (Hall.f.) Stapf & Apocynaceae & Geebbo & $L$ & AD133 \\
\hline 96 & Lepidotrichilia volkensii (Gurke) Leory & Meliaceae & Haalalee & $\mathrm{T}$ & AD138 \\
\hline 97 & Lobelia giberroa Hemsl & Lobeliaceae & Dingiraro & $\mathrm{S}$ & AD169 \\
\hline 98 & Loxogramme abyssinica (Baker) MG. Price & Polypodiaceae & Giixo & $\mathrm{H}$ & AD175 \\
\hline
\end{tabular}


Table 1 (continued)

\begin{tabular}{|c|c|c|c|c|c|}
\hline No & Scientific names & Family & Local names $^{a}$ & Habit & Voucher No. \\
\hline 99 & Macaranga capensis (Baill.) Sim & Euphorbiaceae & Ongo & $\mathrm{T}$ & AD168 \\
\hline 100 & Maesa lanceolata Forssk & Myrsinaceae & Abbayyi & $\mathrm{T}$ & AD027 \\
\hline 101 & Marattia fraxinea Sm & Marattiaceae & - & $\mathrm{H}$ & AD028 \\
\hline 102 & Maytenus gracilipes (Welw.ex Oliv.) Exell & Celastraceae & Kombolcha & S & AD114 \\
\hline 103 & Maytenus undata (Thunb.) Blakelock & Celastraceae & llikke & $\mathrm{T}$ & AD132 \\
\hline 104 & Megalastrum lanuginosum (Willd. ex Kaulf) Holttum & Tectariaceae & - & $\mathrm{H}$ & AD151 \\
\hline 105 & Microglossa pyriflolia (Lam.) O. Kuntze & Asteraceae & Nobbe & $\mathrm{H}$ & AD173 \\
\hline 106 & Millettia ferruginea (Hochst.) Baker & Fabaceae & Sottallo & $\mathrm{T}$ & AD131 \\
\hline 107 & Monothecium glandulosum Hochst & Acanthaceae & Dargu & $\mathrm{H}$ & AD091 \\
\hline 108 & Myrsine africana $\mathrm{L}$ & Myrsinaceae & - & S & AD089 \\
\hline 109 & Ocimum lamiifolium Hochst.ex Benth & Lamiaceae & Damakase & $S$ & AD097 \\
\hline 110 & Olea capensis L & Oleaceae & Gagamaa & $\mathrm{T}$ & AD100 \\
\hline 111 & Olea welwitschii (Knobl.) Gilg \& Schellenb & Oleaceae & Ba'aa & $\mathrm{T}$ & AD050 \\
\hline 112 & Oplismenus hirtellus (L.) P. Beauv & Poaceae & Sutto gogorrii & $\mathrm{H}$ & AD092 \\
\hline 113 & Oxyanthus speciosus DC & Rubiaceae & Abraango jaldessaa & $\mathrm{T} / \mathrm{S}$ & AD079 \\
\hline 114 & Pavonia schimperiana Hochst. ex A. Rich & Malvaceae & Gajjo & $\mathrm{H}$ & AD084 \\
\hline 115 & Pentas schimperiana (A. Rich.) Vatke & Rubiaceae & - & $\mathrm{H}$ & AD031 \\
\hline 116 & Peperomia abyssinica Miq & Piperaceae & Sarxe mukaa & $\mathrm{H}$ & AD176 \\
\hline 117 & Peperomia retusa (L.f.) A. Dietr & Piperaceae & - & $\mathrm{H}$ & AD130 \\
\hline 118 & Peponium vogelii (Hook.f.) Engl & Cucurbitaceae & Tojjo & $\mathrm{H}$ & AD066 \\
\hline 119 & Phaulopsis imbricata (Forssk.) Sweet & Acanthaceae & Dargu & $\mathrm{H}$ & AD039 \\
\hline 120 & Phoenix reclinata Jacq & Araceae & Mexxi & $\mathrm{T}$ & AD022 \\
\hline 121 & Phyllanthus sepialis Muell. Arg & Euphorbiaceae & Qacamaa & S & AD172 \\
\hline 122 & Pilea rivularis Wedd & Urticaceae & - & $\mathrm{H}$ & AD153 \\
\hline 123 & Piper capense L.f & Piperaceae & Tunjo & $\mathrm{H}$ & AD014 \\
\hline 124 & Pittosporum viridiflorum Sims & Pittosporaceae & Soolee & $\mathrm{T}$ & AD070 \\
\hline 125 & Polyscias farinosa (Del.) Harms & Araliaceae & - & $\mathrm{T}$ & AD095 \\
\hline 126 & Polyscias fulva (Hiern) Harms & Araliaceae & Karaso & $\mathrm{T}$ & AD119 \\
\hline 127 & Polystachya rivae Shweinf & Orchidaceae & Capho & $\mathrm{H}$ & AD094 \\
\hline 128 & Polystichum wilsonii $\mathrm{H}$. Christ & Dryopteridaceae & - & $\mathrm{H}$ & AD113 \\
\hline 129 & Pouteria adolfi-friederici (Engl.) Baehni & Sapotaceae & Qararo & $\mathrm{T}$ & AD152 \\
\hline 130 & Premna schimperi Engl & Verbenaceae & Urgessaa & S & AD178 \\
\hline 131 & Prunus africana (Hook. f.) Kalkm & Roseaceae & Homii & $\mathrm{T}$ & AD159 \\
\hline 132 & Psychotria orophila Petit & Rubiaceae & Xumaane & S & AD025 \\
\hline 133 & Pteris dentata Forssk & Pteridaceae & Giixoo & $\mathrm{H}$ & AD157 \\
\hline 134 & Pteris pteridioides (Hook.) ballard & Pteridaceae & Giixoo & $\mathrm{H}$ & AD076 \\
\hline 135 & Pterolobium stellatum (Forssk.) Brenan & Fabaceae & Harangamaa & S & AD154 \\
\hline 136 & Pupalia micrantha Haumam & Amaranthaceae & Maxxanne & $\mathrm{H}$ & AD128 \\
\hline 137 & Ranunculus multifidus Forssk & Ranunculaceae & - & $\mathrm{H}$ & AD149 \\
\hline 138 & Rhamnus prinoides L'Herit & Rhamnaceae & Gesho & S & AD067 \\
\hline 139 & Ritchiea albersii Gilg & Capparidaceae & Daqqo & $\mathrm{T}$ & AD140 \\
\hline 140 & Rothmannia urcelliformis (Hiern) Robyns & Rubiaceae & Diibo & $\mathrm{T}$ & AD069 \\
\hline 141 & Rubus apetalus Poir & Roseaceae & Goraa & $S$ & AD075 \\
\hline 142 & Rubus steudneri Schweinf & Roseaceae & Goraa & S & AD071 \\
\hline 143 & Rytigynia neglecta (Hirn) Robyns & Rubiaceae & Mixo & S & AD112 \\
\hline 144 & Sapium ellipticum (Krauss) Pax & Euphorbiaceae & Bosoqa & $\mathrm{T}$ & AD109 \\
\hline 145 & Scadoxus nutans (Friis \& J. Bjørnstad) Friis \& Nordal & Amaryllidaceae & Qulubi jaldessaa & $\mathrm{H}$ & AD088 \\
\hline 146 & Schefflera abyssinica (Hochst. ex A. Rich.) Harms & Araliaceae & Gatamaa & $\mathrm{T}$ & AD104 \\
\hline 147 & Schefflera myriantha (Bak.) Drake & Araliaceae & Qero & $L$ & AD086 \\
\hline
\end{tabular}


Table 1 (continued)

\begin{tabular}{|c|c|c|c|c|c|}
\hline No & Scientific names & Family & Local names ${ }^{a}$ & Habit & Voucher No. \\
\hline 148 & Sericostachys scandens Gilg \& Lopr & Amaranthaceae & Suddi & $L$ & AD106 \\
\hline 149 & Setaria megaphylla (Steud.) Th. Dur. \& Schinz & Poaceae & Gowaa & $\mathrm{H}$ & AD058 \\
\hline 150 & Solanaceo manni (Hook.f.) C. Jeffrey & Asteraceae & Rejjii caakkaa & $\mathrm{S}$ & AD125 \\
\hline 151 & Solanecio gigas (Vatke) C. Jeffrey & Asteraceae & Raafu boyye & S & AD054 \\
\hline 152 & Solanum adoense Hochst. ex A. Rich & Solanaceae & Hiddi- xino & S & AD102 \\
\hline 153 & Solanum giganteum Jacq & Solanaceae & Tambo arbaa & S & AD048 \\
\hline 154 & Stellaria mannii Hook.f & Caryophyllaceae & Moccoo & $\mathrm{H}$ & AD127 \\
\hline 155 & Syzygium guineense (Willd.) DC & Myrtaceae & Baddessaa & $\mathrm{T}$ & AD141 \\
\hline 156 & Tacazzea apiculata Oliv & Asclepidiaceae & Gebbo & $L$ & AD116 \\
\hline 157 & Tacazzea conferta N.E. Br & Asclepidiaceae & Gebbo qalame & $L$ & AD145 \\
\hline 158 & Teclea nobilis Del & Rutaceae & Mola'ee & $\mathrm{T}$ & AD158 \\
\hline 159 & Tectaria gemmifera (Fee) Alston & Tectariaceae & Gixoo & $\mathrm{H}$ & AD023 \\
\hline 160 & Thalictrum rhynchocarpum Dill. \& A. Rich & Ranunculaceae & Finge & $\mathrm{H}$ & AD105 \\
\hline 161 & Thunbergia alata Boj. ex Sims & Acanthaceae & - & $\mathrm{H}$ & AD043 \\
\hline 162 & Tiliacora troupinii Cufod & Menispermaceae & Liqixi & $L$ & AD146 \\
\hline 163 & Trema orientalis (L.) Bl & Ulmaceae & Huddu farddaa & $\mathrm{T}$ & AD164 \\
\hline 164 & Trichilia dregeana Sond & Meliaceae & Luyyaa & $\mathrm{T}$ & AD049 \\
\hline 165 & Trifolium rueppellianum Fresen & Fabaceae & Amagixa & $\mathrm{H}$ & AD150 \\
\hline 166 & Trilepisium madagascariense DC & Moraceae & Same'eko/ceeyii & $\mathrm{T}$ & AD085 \\
\hline 167 & Tristemma mauritianum J. F. Gmel & Melistostomaceae & - & $\mathrm{H}$ & AD052 \\
\hline 168 & Triumfetta brachyceras K. Schum & Tilaceae & Incciinii & S & AD142 \\
\hline 169 & Turraea holstii Gurke & Meliaceae & Ceekaa & S & AD003 \\
\hline 170 & Urera hypselodendron (A. Rich.) Wedd & Urticaceae & Capho & $L$ & AD047 \\
\hline 171 & Urtica simensis Steudel & Urticaceae & Doobbii & $\mathrm{H}$ & AD115 \\
\hline 172 & Vangueria apiculata K. Schum & Rubiaceae & - & $\mathrm{T}$ & AD056 \\
\hline 173 & Vepris dainellii (Pichi-Serm.) Kokwaro & Rutaceae & Hadhessa & $\mathrm{T}$ & AD098 \\
\hline 174 & Vernonia amygdalina Del & Asteraceae & Eebicha & $\mathrm{T}$ & AD055 \\
\hline 175 & Vernonia auriculifera Hiern & Asteraceae & Rejjii & $\mathrm{T} / \mathrm{S}$ & AD156 \\
\hline 176 & Vernonia hochstetteri Sch. Bip. ex Walp & Asteraceae & Soyama masango & S & AD057 \\
\hline 177 & Vernonia rueppellii Sch. Bip. ex Walp & Asteraceae & Tambo Arbaa & $S$ & AD103 \\
\hline 178 & Vernonia wollastonii S. Moore & Asteraceae & - & $\mathrm{H}$ & AD060 \\
\hline 179 & Zehneria minutiflora (Cogn) C. Jeffrey & Cucurbitaceae & Kalaalaa bosonu & $\mathrm{H}$ & AD063 \\
\hline 180 & Zehneria scabra (Linn. f) Sond & Cucurbitaceae & Kalaalaa bosonu & $\mathrm{H}$ & AD081 \\
\hline
\end{tabular}

${ }^{\mathrm{a}}$ Local name $=$ Afan Oromo

first axis i.e., $<3(2.22)$ which indicate the presence of lower species turnover or homogeneous vegetation data due to the linear relationship between species and environmental variables. The result of Monte Carlo test showed that out of 14 environmental variables, seven were found to be significant in explaining patterns of plant community distribution. From the seven significant environmental factors, the vif values of sand and silt were higher than 5. Sand and Silt are highly correlated with at least one of the other variables in the model. One solution in dealing with collinearity is to remove some of the violating variables from the model and thus the one with higher vif value (sand) was eliminated. The result of RDA ordination showed that comparatively, the gradient of altitude and potassium was highly correlated on axis one and gradient of disturbance in axis two. The other factors were correlated with the five axes with a different value of correlation. The eigenvalue for axis one, two and three were $10.65,8.06$, and 6.32 respectively. Cumulative proportion variance explained by the first five RDA axis of the joint biplot was $93.9 \%$. The proportion of variation explained by five RDA axis also shows a decline towards the successive higher axis (Table 6).

RDA ordination of the study plots of Gerba Dima forest formed five groups or community based on the species composition. These five community types were segregated following the arrows of the environmental variables. Community 3 and community 4 are found in 
Table 2 Endemic species, their habit, IUCN status and geographical distributions

\begin{tabular}{|c|c|c|c|c|}
\hline Species & Family & Habit & IUCN category & Altitude (m) \\
\hline Acanthopale ethio germanica & Acanthaceae & Shrub & $\mathrm{NE}$ & 2300_2600 \\
\hline Aframomum corrorima & Zingiberaceae & Herb & $\mathrm{NE}$ & 1350_2000 \\
\hline Arisaema mooneyanum & Araceae & Herb & $\mathrm{NE}$ & 2000_3450 \\
\hline Bothriocline schimperi & Asteraceae & Shrub & LC & 1300_2820 \\
\hline Clematis longicaudata & Ranunculaceae & Liana & LC & 1350_3300 \\
\hline Crotalaria rosenii & Fabaceae & Herb & NT & 1350_2800 \\
\hline Justicia bizuneshiae & Acanthaceae & Herb & $\mathrm{NE}$ & 1200_2100 \\
\hline Millettia ferruginea & Fabacae & Tree & LC & 1000_2500 \\
\hline Polyscias farinosa & Araliaceae & Tree & VU & 1600_2200 \\
\hline Scadoxus nutans & Amaryllidaceae & Herb & $\mathrm{NE}$ & 1450_2300 \\
\hline Solanecio gigas & Asteraceae & Shrub & LC & 1750_3350 \\
\hline Tiliacora troupinii & Menispermaceae & Liana & $\mathrm{NE}$ & 1500_2100 \\
\hline Urtica simensis & Urticaceae & Herb & LC & 1500_3400 \\
\hline Vepris dainellii & Rutaceae & Ttree & LC & 1750_2500 \\
\hline Vernonia rueppellii & Asteraceae & Shrub & LC & 2150_3000 \\
\hline
\end{tabular}

Source: $[24-32,41]$ LC, Least Concern = A taxon is Least Concern when it has been evaluated against the criteria and does not qualify for Critically Endangered, Endangered, Vulnerable or Near Threatened; NE, Not evaluated = A taxon is Not Evaluated when it is has not yet been evaluated against the criteria; NT, Near Threatened=A taxon is Near Threatened when it has been evaluated against the criteria but does not qualify for Critically Endangered, Endangered or Vulnerable now, but is close to qualifying for or is likely to qualify for a threatened category in the near future; VU, Vulnerable = A taxon is Vulnerable when the best available evidence indicates that it meets any of the criteria, and it is therefore considered to be facing a high risk of extinction in the wild

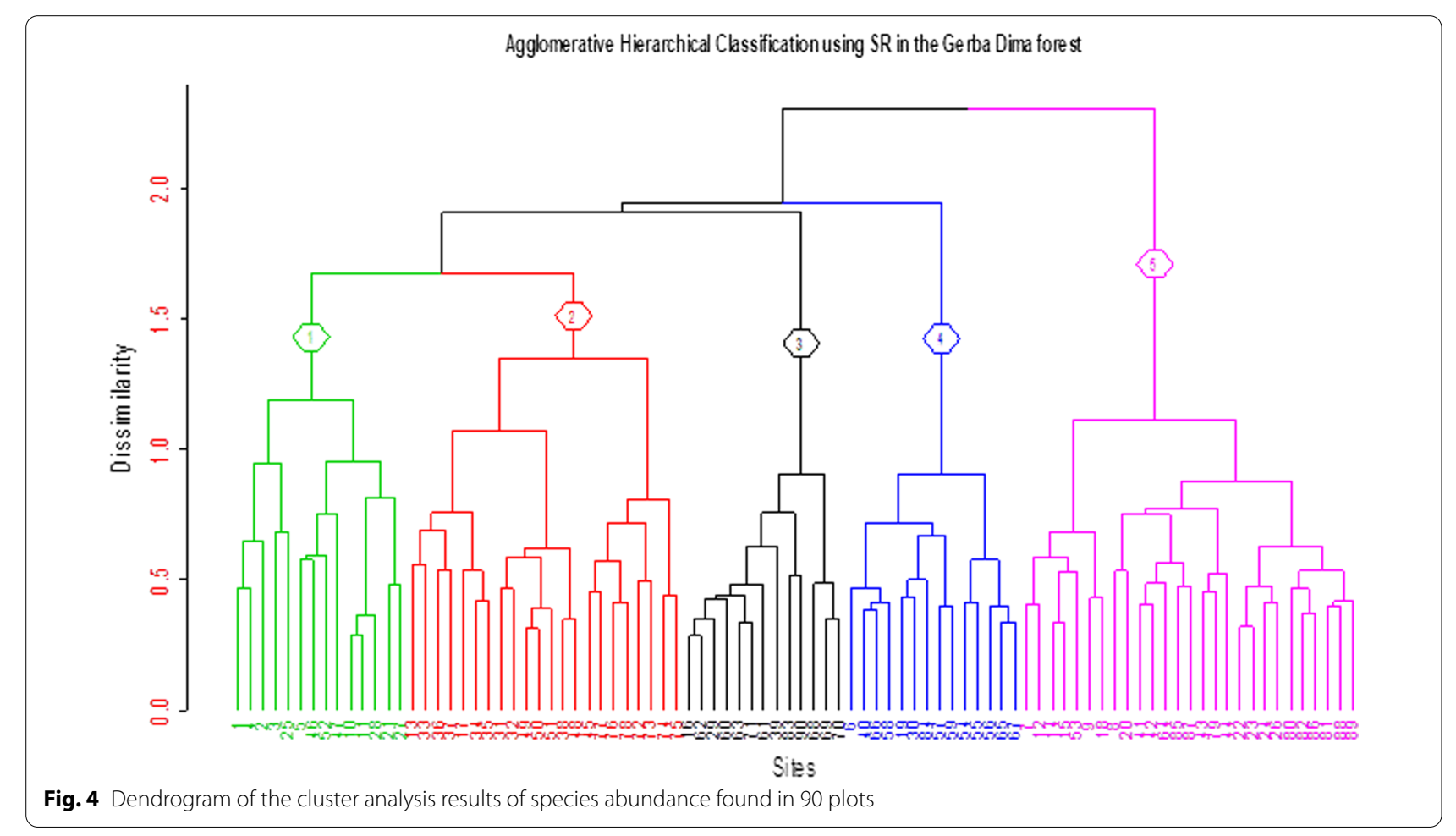

mid altitude area. Community two mostly occur at the higher altitude while species in community 1 and community 5 are distributed at the lower altitude and higher EC. Silt, Disturbance and potassium axes were strongly influencing the distribution of community five. Organic matter arrow has strongly influenced the distribution of species in community three and four (Fig. 5). The ANOVA test indicated that the five community types 
differ significantly from each other with regard to EC and $\mathrm{K}$. The result of Tukey's pair-wise comparison test indicates that community 4 and 1 differ significantly with respect to Disturbance and K while community 2 and 3 showed significant differences with respect to EC.

Table 3 Synoptic cover value of plant in Gerba Dima Forest for species reaching $\geq 1 \%$ in at least one community

\begin{tabular}{|c|c|c|c|c|c|}
\hline Cluster number & C1 & $\mathrm{C} 2$ & $\mathrm{C} 3$ & C 4 & C5 \\
\hline Cluster size & 14 & 22 & 13 & 14 & 27 \\
\hline Allophyllus abyssinicus & 3.50 & 1.05 & 1.15 & 0.86 & 1.26 \\
\hline Bersama abyssinica & 3.71 & 1.73 & 0.08 & 0.64 & 1.22 \\
\hline Croton macrostachyus & 7.50 & 1.77 & 1.54 & 1.79 & 2.48 \\
\hline Cordia africana & 2.79 & 0.55 & 0.00 & 0.00 & 0.63 \\
\hline Olea welwitschii & 1.43 & 1.27 & 0.15 & 0.86 & 0.56 \\
\hline Ehretia cymosa & 2.36 & 2.55 & 1.92 & 0.79 & 1.63 \\
\hline Polyscias fulva & 1.64 & 1.36 & 1.85 & 0.93 & 1.33 \\
\hline Apodytes dimidiate & 1.43 & 2.41 & 1.54 & 1.64 & 0.93 \\
\hline Olea capensis & 3.14 & 5.59 & 1.54 & 1.93 & 1.04 \\
\hline Syzygium guineense & 0.64 & 5.91 & 1.69 & 2.43 & 1.56 \\
\hline Justicia schimperiana & 1.29 & 1.68 & 0.85 & 0.36 & 0.93 \\
\hline Canthium oligocarpum & 0.29 & 1.05 & 0.62 & 0.50 & 0.52 \\
\hline Cassipourea malosana & 0.79 & 1.55 & 1.08 & 0.43 & 0.56 \\
\hline Combretum paniculatum & 0.86 & 1.14 & 0.54 & 0.43 & 1.04 \\
\hline Dracaena steudneri & 1.29 & 3.09 & 0.92 & 1.50 & 1.22 \\
\hline Elaeodendron buchananii & 0.64 & 1.00 & 0.38 & 0.00 & 0.37 \\
\hline Oplismenus hirtellus & 2.43 & 4.50 & 3.00 & 2.64 & 2.85 \\
\hline Rothmannia urcelliformis & 1.14 & 1.95 & 0.77 & 0.86 & 1.15 \\
\hline Sapium ellipticum & 0.21 & 1.64 & 0.00 & 1.43 & 0.93 \\
\hline Tectaria gemmifera & 0.93 & 1.36 & 1.23 & 1.29 & 0.81 \\
\hline Brillantaisia madagascariensis & 1.43 & 2.73 & 3.00 & 2.79 & 2.48 \\
\hline Dracaena afromontana & 1.00 & 3.05 & 7.69 & 0.86 & 0.78 \\
\hline Ficus sur & 2.50 & 1.68 & 6.77 & 2.07 & 1.37 \\
\hline Galiniera saxifrage & 1.00 & 1.05 & 2.31 & 1.43 & 1.56 \\
\hline Hallea rubrostipulata & 1.07 & 0.00 & 1.31 & 0.00 & 0.00 \\
\hline Macaranga capensis & 1.21 & 1.32 & 2.85 & 0.71 & 0.19 \\
\hline Oxyanthus speciosus & 1.29 & 1.73 & 7.01 & 2.36 & 1.56 \\
\hline Pouteria adolf-friederici & 2.21 & 3.05 & 7.31 & 2.07 & 1.26 \\
\hline Acanthopale ethio-germanica & 1.36 & 0.77 & 2.08 & 2.43 & 1.96 \\
\hline Deinbollia kilimandscharica & 0.57 & 1.45 & 2.62 & 4.07 & 1.59 \\
\hline Ilex mitis & 0.43 & 0.59 & 1.31 & 4.71 & 0.44 \\
\hline Justicia bizuneshiae & 0.50 & 1.23 & 1.31 & 1.71 & 1.37 \\
\hline Landolphia buchananii & 1.00 & 1.32 & 0.85 & 1.43 & 1.19 \\
\hline Piper capense & 1.00 & 0.55 & 0.46 & 1.43 & 1.07 \\
\hline Psychotria orophila & 0.93 & 1.36 & 0.85 & 1.36 & 0.85 \\
\hline Pupalia micrantha & 0.64 & 1.36 & 0.23 & 1.86 & 0.93 \\
\hline Schefflera abyssinica & 0.50 & 1.73 & 1.38 & 7.29 & 1.33 \\
\hline Tiliacora troupinii & 1.00 & 1.23 & 1.08 & 1.29 & 1.07 \\
\hline Vepris dainellii & 2.21 & 3.36 & 3.00 & 8.43 & 3.59 \\
\hline Albizia gummifera & 3.07 & 2.50 & 2.69 & 2.07 & 8.63 \\
\hline Clausena anisate & 1.79 & 1.86 & 1.31 & 1.43 & 2.11 \\
\hline Hippocratea pallens & 0.64 & 1.91 & 1.23 & 1.50 & 1.93 \\
\hline
\end{tabular}

Table 3 (continued)

\begin{tabular}{llllll}
\hline Cluster number & C1 & C 2 & C 3 & C 4 & C 5 \\
\hline Lepidotrichilia volkensii & 0.57 & 2.59 & 1.23 & 2.43 & 3.30 \\
Maytenus gracilipes & 2.00 & 1.82 & 1.08 & 2.00 & 2.30 \\
Millettia ferruginea & 2.00 & 2.95 & 2.54 & 2.79 & $\mathbf{7 . 8 9}$
\end{tabular}

$\mathrm{C} 1$, Croton macrostachyus-Bersama abyssinica; C2, Syzygium guineense-Olea capensis; C3, Dracaena afromontana -Pouteria adolf-friederici; C4, Vepris dainellii Schefflera abyssinica C5, Albizia gummifera-Millettia ferruginea community. Values in bold indicate the synoptic value of dominant species used in naming the plant communities

Table 4 Indicator species of clusters in Gerba Dima forest with their significant P-value

\begin{tabular}{llll}
\hline Name of indicator species & $\begin{array}{l}\text { Community } \\
\text { type (C) }\end{array}$ & Indicator value & P-value \\
\hline Prunus Africana & 1 & 0.528 & $0.018^{*}$ \\
Rubus apetalus & 1 & 0.516 & $0.017^{*}$ \\
Flacourtia indica & 2 & 0.521 & $0.02^{*}$ \\
Pilea rivularis & 3 & 0.498 & $0.016^{*}$ \\
Elastostema monticolum & 3 & 0.467 & $0.039^{*}$ \\
Ritchiea albersii & 4 & 0.861 & $0.001^{* * *}$ \\
Trema orientalis & 4 & 0.677 & $0.001^{* * *}$ \\
Sapium ellipticum & 4 & 0.636 & $0.002^{* *}$ \\
Vernonia hochstetteri & 4 & 0.538 & $0.014^{*}$ \\
Zehneria scabra & 5 & 0.581 & $0.002^{* *}$ \\
Zehneria minutiflora & 5 & 0.552 & $0.005^{* *}$ \\
Urera hypselodendron & 5 & 0.478 & $0.017^{*}$ \\
Vernonia wollastonii & 5 & 0.423 & $0.045^{*}$
\end{tabular}

C1, Croton macrostachyus-Bersama abyssinica; C2, Syzygium guineense-Olea capensis; C3, Dracaena afromontana-Pouteria adolfi-friederici; C4, Vepris dainellii Schefflera abyssinica $C 5$, Albizia gummifera-Millettia ferruginea community. ${ }^{*}=(p<0.5),{ }^{* *}=(p<0.01),{ }^{* *}=(p<0.001)$

Table 5 Species richness, evenness and diversity indices of plant community types

\begin{tabular}{llll}
\hline Community & $\begin{array}{l}\text { Species } \\
\text { richness }\end{array}$ & $\begin{array}{l}\text { Shannon diversity } \\
\text { index }\left(\mathbf{H}^{\prime}\right)\end{array}$ & $\begin{array}{l}\text { Shannon } \\
\text { Evenness }\end{array}$ \\
\hline 1 & 138 & 4.40 & 0.89 \\
2 & 144 & 4.27 & 0.86 \\
3 & 107 & 3.99 & 0.85 \\
4 & 104 & 4.05 & 0.87 \\
5 & 140 & 4.19 & 0.85 \\
\hline
\end{tabular}

\section{Discussion}

Floristic composition and diversity of Gerba Dima forest The existence of diversified flora of Gerba Dima forest was in line with the general pattern of high species diversity in the tropical montane forests. According to Gentry 


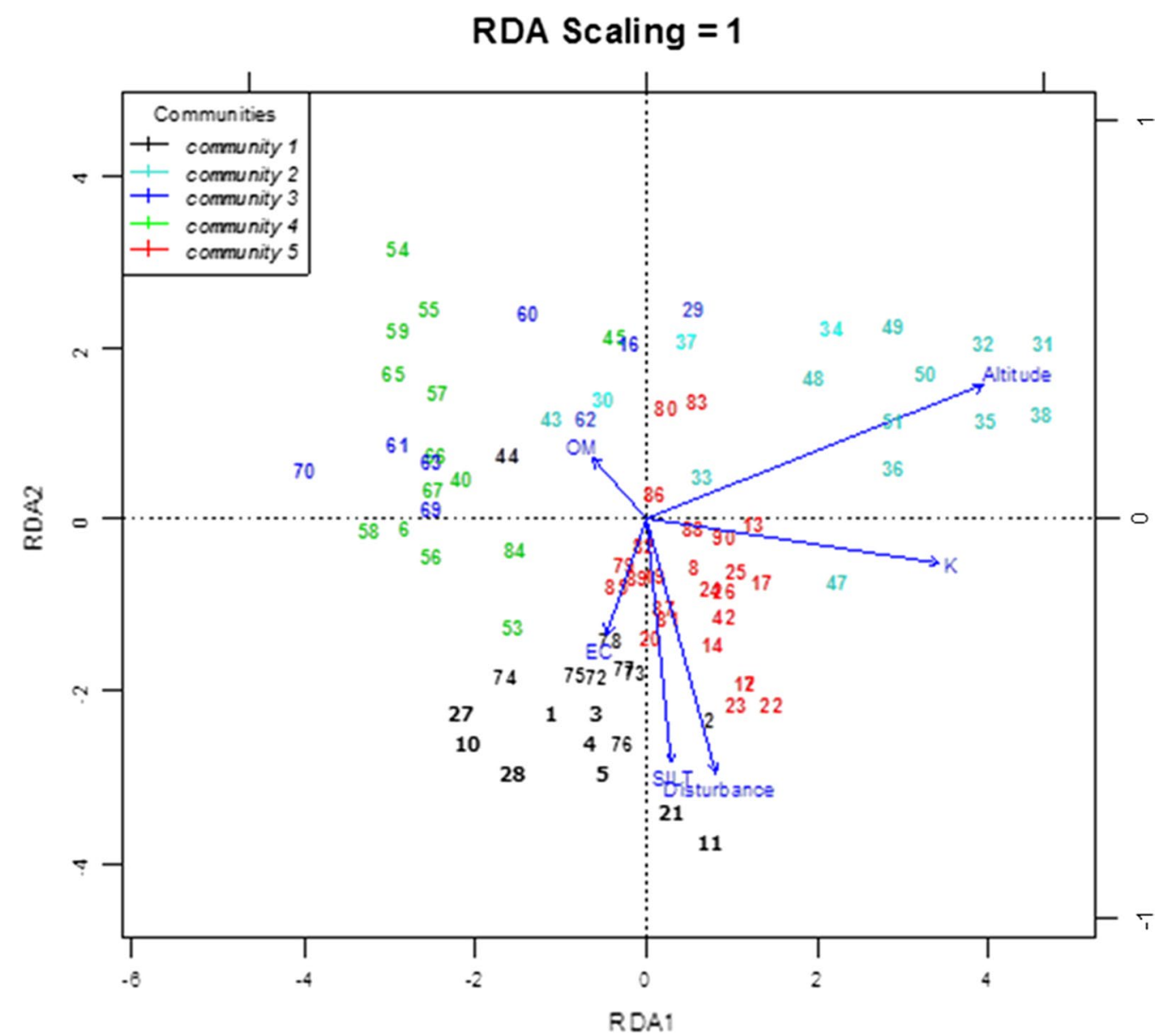

Fig. 5 RDA ordination biplot of 90 quadrats and 6 environmental variables of plant communities

Table 6 Biplot score for constraining variables and their correlation with the RDA axis, eigenvalues and proportion of variance explained

\begin{tabular}{lrrrrr}
\hline $\begin{array}{l}\text { Environmental } \\
\text { variables }\end{array}$ & RDA1 & RDA2 & RDA3 & RDA4 & RDA5 \\
\hline Disturbance & 0.089 & -0.68 & -0.157 & 0.522 & 0.453 \\
Altitude & 0.880 & 0.42 & -0.054 & -0.018 & 0.218 \\
SILT & 0.084 & -0.40 & -0.361 & -0.323 & -0.370 \\
EC & -0.053 & 0.31 & -0.867 & 0.094 & -0.023 \\
OM & -0.094 & 0.27 & -0.208 & -0.357 & 0.844 \\
K & 0.703 & -0.27 & -0.003 & -0.381 & -0.246 \\
Eigenvalue & 10.6445 & 8.0649 & 6.3168 & 5.0057 & 3.02318 \\
Proportion explained & 0.3024 & 0.2291 & 0.1794 & 0.1422 & 0.08588 \\
Cumulative proportion & 0.3024 & 0.5315 & 0.7109 & 0.8531 & 0.93902 \\
\hline
\end{tabular}

[42], tropical forests are among ecosystems that harbour high species diversity of the globe. East African montane forests of Ethiopia, Kenya, Tanzania and Uganda are among the most diverse and richest African regions with regard to flora composition and endemic plant taxa [43-45]. Asteraceae, Acanthaceae, Rubiaceae, Fabaceae and Euphorbiaceae are the five dominant families, which contribute more than $27 \%$ of the total species in the study forest. These dominant families were also reported as top ten species rich families in many Neotropical forests and Asia [42]. Except for Rubiaceae, these families are also among the top ten species rich families in the flora area [46]. The dominance of the above families together with Rubiaceae was also reported in other moist afromontane forests of southwestern Ethiopia [47-49]. Thus, the dominance of these families in the Gerba Dima forest agreed to their general dominance in the flora area and tropical forests. The dominance of these families in the study area could be attributed to their successful colonization to the landscape owing to their efficient pollination, dispersal and germination mechanisms [50]. For instance, many species of Asteraceae have umbrella shape structures adapted for air dispersal and increase their opportunity for their successful establishment [50]. 
Among the growth forms, herbs constitute more than $42 \%$ of recorded species. The prevalence of herbs could be attributed to the presence of canopy gap because of anthropogenic disturbance. Disturbance of forest in the form of selective cutting of trees favours the growth of herbaceous species in the forest understory. Under normal circumstances, the forest floor (herbaceous layer) of Afromontane rainforests is usually dark and poor in species composition owing to the closed canopy of the forest that prevents light from reaching the ground [51].

The higher value of Shannon diversity index and evenness indicates that the study forest has high species diversity with more even distribution of the species within the study plots. Species diversity increases when the populations have more even abundances and vice versa [40]. High Shannon evenness in the Gerba Dima forest indicates little dominance by any single species but the repeated coexistence of species over all the plots or sites. Therefore, the implication of evenness values is that, when there is a high evenness value in a given forest, the location of conservation sites might not be of much importance compared to when the evenness value of the forest is low.

To give a general impression of the species richness of Gerba Dima Forest, the results of the present study were compared with results from other Moist Afromontane forests in Ethiopia. The species richness of Gerba Dima forest is higher than some moist afromontane forest of Ethiopia such as Masha forest (130 species) [48], Belete forest (157 species) [52], Gelesha forest (157 species) [53], Agama forest (162 species) [49] and more or less similar in species richness with some other moist afromontane forest of Ethiopia such as Komto forest (180 species) [54] and Jibat forest (183 species) [55]. However, the species richness of Gerba Dima forest was much lower than the values reported for few other moist afromontane forest of Ethiopia which include Bonga forest (243 species) [47], Yayu forest (220 species) [56] Mana Angetu forest (212 species) [57] (Magada forest (197 species) [58] and Gesha and Sayilem forest (300 species [59].

The difference in species richness among the compared forests could be attributed to the variations of forest sites with regard to geographical location, altitude, anthropogenic impact, rainfall and other climatic, physiographic and edaphic factors $[60,61]$. Climatic and physiographic factors have a wide range of effect on the diversity of plant species across the land escape whereas suitable environmental conditions and biotic factors influence diversity at the site level $[62,63]$. Species composition of forests is also influenced by regeneration success and competition among species [64].

\section{Plant community types in Gerba Dima forest}

The output of Multi-response Permutation Procedures (MRPP) results in $\mathrm{T}$ statistics having more negative value with significant $\mathrm{P}$-value $(\mathrm{T}=-38.26, \mathrm{P}<0.001)$ and an agreement statistic A (0.13) confirming the distinctness of clusters. The test statistic $\mathrm{T}$ describes the separation between the groups. The more negative $T$ value, the stronger the separation. From the result of this study, the null hypothesis of no difference among groups can be rejected. The five groups occupy different regions of species space, as shown by the strong chance correction within the group (A) and test statistic $(\mathrm{T})$ and thus confirm the existence of 5 distinct plant communities in the Gerba Dima forest [37]. The five plant communities showed a slight variation in their species richness, diversity and evenness. Relatively community types 1,2 and 5 were the richest with respect to species richness and diversity while community types 3 and 4 the lowest. The differences in species richness among the five communities could mainly be attributed to the dissimilarities of the communities in terms of location, altitude, human impact, rainfall, and other biotic and abiotic factors. According Eilu and Obua to [65], different altitudes and slopes influence species richness and dispersion behaviour of tree species. Altitude and climatic variables like temperature and rainfall are also other determinant factors that affect species richness [66].

\section{Plant community-environmental variables relationship}

In the current study, the multivariate analyses (both Ordination and cluster analysis) were consistent in showing the patterns of floristic grouping within the studied forest and hence the two methods are complementary. The variable with the highest score $(0.88)$ associated with axis one was the altitude. Therefore, altitude was the most important variable in weighting axis one and to interpret or explain the axis. Similar studies conducted in other Afromontane forests of Ethiopia also confirm the importance of altitude as a major determinant of vegetation distribution along altitudinal gradients $[57,67,68]$. Altitudinal change leads to changes in humidity, temperature, soil type, and other factors that influence the growth and development of plants which in turn determine the patterns of vegetation distribution $[69,70]$.

Potassium followed by altitude was also the most important constraining variable in weighing axis one in the ordination. In the sandy soil, plant-soil feedback effects were most strongly correlated with potassium. Although most studies investigating abiotic plant-soil interactions have focused on nitrogen and phosphorus dynamics, in sandy soils with little clay content, potassium could be a limiting factor for plant growth [71, 72]. In particular, a growth of forbs can be highly dependent 
on potassium [71] and hence potassium at least affects the distribution of these species. In the same way, the disturbance was the most important variable in weighting axis two. Disturbance affects the distribution of plant communities by hampering natural regeneration and seedling establishment in tropical forests [73]. Disturbance also favours the growth of herbaceous plant species by improving the availability of light conditions in the ground layer as it widens the canopy gap [74] and thus affects the distribution of communities with these species. An analysis of variance (ANOVA) performed to see any significant variation among the community types of Gerba Dima forest with respect to non-collinear significant environmental variables indicated that the five community types differ significantly from each other with regard to EC and K. Similarly, result of Tukey's pairwise comparison test indicates that community 4 and 1 differ significantly with respect to Disturbance and $\mathrm{K}$ while community 2 and 3 showed significant difference with respect to EC.

\section{Conclusions}

Description of the floristic diversity of species in the Gerba Dima forest revealed the presence of high species diversity and richness. Of the species recorded in this forest, 15 (8.3\%) species were endemic to Ethiopia. However, the percentage of endemic species in the study forest is lower than the proportions generally expected in the Afromontane forest of Ethiopia and this is attributed to the low endemicity feature of forests in South-western Ethiopia. In this study, five community types were identified and altitude was the major environmental variable in determining the community types. The existence of high species diversity and a number of endemic plant species in the study forest shows the potential of the area for biodiversity conservation. Thus, all Stakeholders including Oromia Forest and wildlife enterprise (OFWE) and the regional government should work to designate the forest as a biosphere reserve and being registered under UNESCO.

\section{Abbreviations}

DCA: Detrended Correspondence Analysis; RDA: Redundancy Analysis; Vif: Variance inflation factor; MRPP: Multi-response Permutation Procedures.

\section{Supplementary Information}

The online version contains supplementary material available at https://doi. org/10.1186/s12862-022-01964-4.

Additional file 1: Dominant families with their respective species number of Gerba Dima Forest.
Acknowledgements

We would like to thank Addis Ababa University for providing logistics for fieldwork and laboratory analysis.

\section{Authors' contributions}

All authors have made substantive intellectual contributions to this manuscript. AD is made substantial contributions to conception and design, or acquisition of data, or analysis and interpretation of data and also been involved in drafting the manuscript or revising it critically for important intellectual content. TS is also made substantial contributions to conception and design of data, or analysis and interpretation of data but not involved in data collection or acquisition of data. BW has been involved in drafting the manuscript or revising it critically for important intellectual content. All authors read and approved the final manuscript.

\section{Funding}

This study was supported by International Foundation for Science (Grant Number D/5481-1). The funders had no role in study design, data collection and interpretation, or the decision to submit the work for publication.

\section{Availability of data and materials}

We have also included part of the data used in this research and attached as Additional files 1.

\section{Declarations}

\section{Ethics approval and consent to participate}

This research is mainly an ecological and study and did not involve experiment on plant species. Thus, Parts related to Ethics approval and consent to participate is not applicable for this work. Related to this part, the collected plant specimens in this research were deposited in the national herbarium of Ethiopia. However, experiment was not conducted on the plant. Permissions were needed and subsequently obtained From Oromia Forest and Wildlife Enterprise in order to use/sample the land as described, and obtain samples.

\section{Consent for publication}

Not applicable.

\section{Competing interests}

The authors declare that they have no competing interests.

\section{Author details}

${ }^{1}$ College of Natural Sciences, Department of Biology, Debre Berhan University, P. O. Box 445, Debre Berhan, Ethiopia. ${ }^{2}$ Center for Environmental Science, Addis Ababa University, P. O. Box 1176, Addis Ababa, Ethiopia. ${ }^{3}$ College of Natural Sciences, Department of Plant Biology and Biodiversity Management, Addis Ababa University, P. O. Box 3434, Addis Ababa, Ethiopia.

Received: 20 October 2021 Accepted: 18 January 2022

Published online: 07 February 2022

\section{References}

1. Kent M. Vegetation description and data analysis: a practical approach. Chichester: Wiley; 2012. p. 414.

2. Legendre P. Spatial autocorrelation: trouble or new paradigm? Ecology. 1993;74:1659-73.

3. Seabloom EW, Bjornstad ON, Bolker BM, Reichman OJ. Spatial signature of environmental heterogeneity, dispersal, and competition in successional grasslands. Ecol Monogr. 2005;75:199-214.

4. Dale MRT. Spatial pattern analysis in plant ecology. Cambridge, UK: Cambridge University Press; 1999.

5. MacArthur R, Levins R. The limiting similarity, convergence, and divergence of coexisting species. Am Nat. 1967;101:377-85.

6. Götzenberger L, de Bello F, Bräthen KA, Davison J, Dubuis A, Guisan A, Lepŝ J, Lindborg R, Moora M, Zobel M. Ecological assembly rules in plant communities - approaches, patterns and prospects. Biol Rev. 2012;87:111-27. 
7. MacArthur R. Geographical ecology: patterns in the distribution of species. Princeton: Princeton University Press; 1972.

8. Keddy P, Weiher E. Introduction: the scope and goals of research on assembly rules. In: Weiher E, Keddy P, editors. Ecological assembly rules: perspectives, advances, retreats. Cambridge: Cambridge University Press; 1999. p. 1-20.

9. Tilman D, Downing JA. Biodiversity and stability in grasslands. Nature. 1994;367:363-5.

10. Grace JB. Examining the relationship between environmental variables and ordination axes using latent variables and structural equation modeling. In: Pugesek BH, Tomer A, von Eye A, editors. Structural equation modeling: applications in ecological and evolutionary biology. Cambridge: Cambridge University Press; 2003. p. 171-93.

11. Kashian DM, Barnes BV, Walker WS. Ecological species groups of landform-level ecosystems dominated by jack pine in northern Lower Michigan, USA. Plant Ecol. 2003;166:75-91.

12. Naumburg E, DeWald LE. Relationships between Pinus ponderosa forest structure, light characteristics, and understory graminoid species presence and abundance. For Ecol Manage. 1999;124:205-15.

13. Laughlin DC, Bakker JD, Fulê PZ. Understorey plant community structure in lower montane and subalpine forests, Grand Canyon National Park. USA J Biogeogr. 2005;32:2083-102.

14. Hayes GF, Holl KD. Cattle grazing impacts on annual forbs and vegetation composition of mesic grasslands in California. Conserv Biol. 2003;17:1694-702.

15. Malik ZA, Bhatt AB. Phytosociological analysis of woody species in Kedarnath Wildlife Sanctuary and its adjoining areas in Western Himalaya, India. J For Environ Sci. 2015;31:149-63.

16. Mishra AK, Behera SK, Singh K, et al. Influence of abiotic factors on community structure of understory vegetation in moist deciduous forests of north India. For Sci Pract. 2013;15:261-73.

17. FAO. Global Forest Resources Assessment 2010-Country Report Ethiopia. Food and Agriculture Organization (FAO), Rome, Italy. 2010.

18. Westpha E. Agricultural system in Ethiopia. Poduc: Wageningen; 1975.

19. Asres T. Agroecological zones of Southwest Ethiopia. Matreialien Zurostafrica Forschung. 1996;13:1-241

20. NMSA. National Meteorological Service Agency, Ethiopia. 2016.

21. Friis I, Demissew $S$, van Breugel P. A new potential vegetation map of Ethiopia in 1:2,000,000. Det Kongelige Danske Videnskabers Selskab. 2010

22. Mueller D, Ellenberg H. Aims and methods of vegetation ecology. New York: Willey; 1974. p. 547.

23. Van der Maarel E. Transformation of cover abundance values in phytosociology and its effects on community. Vegetation. 1979;39:97-114.

24. Edwards S, Tadesse M, Hedberg I, editors. Flora of Ethiopia and Eritrea. Canellaceae to Euphorbiaceae, vol 2(2). Addis Ababa: The National Herbarium, Addis Ababa University. 1995.

25. Edwards S, Demissew S, Hedberg I, editors. Flora of Ethiopia and Eritrea. Hydrocharitaceae to Arecaceae, vol 6. Addis Ababa: The National Herbarium, Addis Ababa University. 1997.

26. Edwards S, Tadesse M, Demissew S, Hedberg I, editors. Flora of Ethiopiaand Eritrea. Magnoliaceae to Flacourtiaceae, vol 2(1). Addis Ababa: The National Herbarium, Addis Ababa University. 2000.

27. Hedberg I, Edwards S, editors. Flora of Ethiopia. Pittosporaceae to Araliacae, vol 3. Addis Ababa: The National Herbarium, Addis Ababa University. 1989.

28. Hedberg I, Edwards S, Nemomissa S, editors. Flora of Ethiopia and Eritrea. Asteraceae, vol 4(2). Addis Ababa: The National Herbarium, Addis Ababa University. 2004.

29. Hedberg I, Kelbessa E, Edwards S, Demissew S, Persson E, editors. Flora of Ethiopia and eritrea. Gentianaceae to Cyclocheilaceae, vol 5. Addis Ababa: The National Herbarium, Addis Ababa University. 2006.

30. Hedberg I, Edwards S, Nemomissa S, editors. Flora of Ethiopia and Eritrea. Apiaceae to Dipsaceae, vol 4(1). Addis Ababa: The National Herbarium, Addis Ababa University. 2003.

31. Hedberg I, Edwards S, editors. Flora of Ethiopia and Eritrea. Poaceae, vol 7. Addis Ababa: The National Herbarium, Addis Ababa University. 1995.

32. Hedberg I, Friis IB, Persson E, editors. Flora of Ethiopia and Eritrea. Lyvopodiaceae to Pinaceae, vol 1. Addis Ababa: The National Herbarium, Addis Ababa University. 2009.
33. Woldu Z, Feoli E, Nigatu L. Partitioning an elevation gradient of vegetation from south eastern Ethiopia by probabilistic methods. Vegetation. 1989:81:189-98.

34. Anderson EW, Currier WF. Evaluating zones of Utilization. J Range Manag. 1973;26(2):87-91.

35. Allen SE. Chemical analysis of ecological materials. Oxford: Blackwell; 1989.

36. R Development Core Team. R: A Language and environment for statistical computing. 2019. http://www.R-project.org/.

37. McCune B, Grace JB. Analysis of ecological communities. USA: MjM SoftwareDesign; 2002.

38. Woldu Z. Comprehensive analysis of vegetation and ecological data: basics, concepts and methods. Addis Ababa: Addis Ababa University Press; 2016. p. 408.

39. Van der Maarel E, Espejel I, Moreno-Casasola P. Two-step vegetation analysis based on very large data sets. Vegetation. 1987;68:139-43.

40. Magurran AE. Ecological diversity and its measurement. London: Chapman and Hall; 1988

41. Vivero JL, Kelbessa E, Demissew S. The Red List of Endemic Trees \& Shrubs of Ethiopia and Eritrea. Fauna \& Flora International, United Kingdom. 2005; p. 28

42. Gentry AH. Patterns of diversity and floristic composition in neotropical montane forests. In: Biodiversity and Conservation of Neotropical Montane Forests. In: Churchill SP, Balslev H, Forero E, Luteyn JL, eds. The New York Botanical Garden, Bronx, New York. 1995; pp 103-126.

43. Coetzee JA. Phytogeographical aspects of the montane forests of the chain ofmountains on the eastern side of Africa. Erdwissenschaft Forschung. 1978;11:482-94.

44. Bekele T. Vegetation ecology of remnant Afromontane forests on the centralplateau of Shewa, Ethiopia. Acta Phytogeographica, Sukecica. 1993:79:1-59.

45. Lovett JC. Eastern Tropical African Center of Endemism: a candidate for worldheritage status? J East Afr Nat Hist. 1998:87:359-66.

46. Kelbessa E, Demissew S. Diversity of vascular plant taxa of the flora of Ethiopia and Eritrea. Ethiop J Biol Sci. 2014;13(1):37-45.

47. Kelbessa E, Soromessa T. Interfaces of regeneration, structure, diversity anduse of some plant species in Bonga forest: aA reservoir for wild coffee gene pool. SINET Ethiop J Sci. 2008:31(2):121-34.

48. Assefa A, Demissew S, Woldu Z. Floristic composition, structure andregeneration status of Masha forest, south-west Ethiopia. Afr J Ecol. 2013;52:151-62.

49. Addi A, Soromessa T, Kelebessa K, Dibaba A, Kefyalew A. Floristiccomposition and plant community types of Agama Forest, an "Afromontane Forest" inSouthwest Ethiopia. J Ecol Nat Environ. 2016;8(5):55-69.

50. Hedberg O. Features of Afroalpine plant ecology. Acta PhytogeographySuec. 1964:49:1-144.

51. Hedberg I, Friis I, Persson E, editors. Flora of Ethiopia and Eritrea Volume 8, General part and Index to Volumes 1-7. Addis Ababa, Ethiopia and Uppsala, Sweden. 2009.

52. Gebre Hiwot K, Hundera K. Species composition, Plant Community structure and Natural regeneration status of Belete Moist Evergreen Montane Forest, Oromia Regional state, Southwestern Ethiopia. Momona Ethiop J Sci (MEJS). 2014:1:97-101.

53. Alemu B, Kitessa Hundera K, Abera B. Floristic composition and structural analysis of Gelesha forest, Gambella regional State. Southwest Ethiop J Ecol Nat Environ. 2015;7(7):218-27.

54. Gurmessa F, Soromessa T, Kelbessa E. Floristic composition and community analysis of Komto Afromontane moist forest, East Wollega Zone, West Ethiopia. Sci Technol Arts Res J. 2013;2(2):58-69.

55. Burju T, Hundera K, Kelbessa E. Floristic composition and structural analysis of Jibat Humid Afromontane Forest, West Shewa Zone, Oromia National Regional State, Ethiopia. Ethiop J Educ \& Sc. 2013:8(2):11-33.

56. Woldemariam T, Borsch T, Denich M, Teketay D. Floristic composition and environmental factors characterizing coffee forests in southwest Ethiopia. For Ecol Manage. 2008;255:2138-50.

57. Lulekal E, Kelbessa E, Bekele T, Yineger H. Plant species composition and structure of the Mana Angetu Moist Montane Forest, South-Eastern Ethiopia. J East Afr Nat Hist. 2008;97:165-85.

58. Bekele G, Reddy PR. Study of vegetation composition of Magada Forest, Borana Zone, Oromia, Ethiopia. Universal J Plant Sci. 2015:3(5):87-96. 
59. Addi A, Soromessa T, Bekele T. Plant diversity and community analysis of Gesha and Sayilem Forest in Kaffa Zone, southwestern Ethiopia. Biodiversitas. 2020;21:2878-88.

60. Shmida A, Wilson MV. Biological determinants of species diversity. J Biogeogr. 1985;12:1-20.

61. Brockway DG. Forest plant diversity at local and landscape scales in the Cascade Mountains of southwestern Washington. For Ecol Manage. 1998;109:323-41.

62. Pausas JG, Austin MP. Patterns of plant species richness in relation to different environments: an appraisal. J Veg Sci. 2001;12:153-66.

63. Tuomisto H, Roukolainen $\mathrm{K}$, Yli-Halla M. Dispersal, environment, and floristic variation of Western Amazonian Forests. Science. 2003;299:241-4.

64. Chen J, Song B, Rudnicki M, Moeur M, Bible K, North M, Shaw DC, Franklin JF, Braun DM. Spatial relationship of biomass and species distribution in an old-growth Pseudotsuga - Tsuga Forest. For Sci. 2003;50:364-75.

65. Eilu G, Obua J. Tree condition and natural regeneration in disturbed sites of Bwindi Impenetrable forest national park, Southwestern Uganda. Trop Ecol. 2005;46:99-111.

66. Kharkwal G, Mehrotra P, Rawat YS, Pangtey YPS. Phytodiversityand growth form in relation to altitudinal gradient in the Central Himalayan (Kumaun) region of India. Curr Sci. 2005;89:873-8.

67. Yeshitela K, Bekele T. Plant community analysis and ecology of Afromontane and transitional rainforest vegetation of south-western Ethiopia. SINET Ethiop J Sci. 2002;25(2):155-75.

68. Yineger $\mathrm{H}$, Kelbessa $\mathrm{E}$, Bekele T, Lulekal E. Floristic composition and structure of the dry Afromontane Forest at Bale Mountains National Park. SINET Ethiop J Sci. 2008;31(2):103-20.

69. Austin M, Pausas J, Nicholls A. Patterns of tree species richness in relationto environment in South-eastern New South Wales, Australia. Austra J Ecol. 1996;21:164-5.

70. Vittoz P, Bayfield N, Brooker R, Elston DA, Duff El, Theurillat JP, Guisan A. Reproducibility of species lists, visual cover estimates and frequency methods for recording high-mountain vegetation. J Veg Sci. 2010;21:1035-47.

71. Tilman EA, Tilman D, Crawley MJ, Johnston AE. Biological weed control via nutrient competition: potassium limitation of dandelions. Ecol Appl. 1999:9:103-11.

72. Kayser M, Isselstein J. Potassium cycling and losses in grassland systems: a review. Grass Forage Sci. 2005;60:213-24.

73. Bussmann RW. Succession and regeneration patterns of East African mountain forests. Syst Geogr Plants. 2001:71:957-74.

74. Engelbrecht BMJ, Comita LS, Condit R, Kursar TA, Tyree MT, Turner BL, Hubbell SP. Drought sensitivity shapes species distribution patterns in tropical forests. Nature. 2007;447(3):80-3.

\section{Publisher's Note}

Springer Nature remains neutral with regard to jurisdictional claims in published maps and institutional affiliations.

Ready to submit your research? Choose BMC and benefit from:

- fast, convenient online submission

- thorough peer review by experienced researchers in your field

- rapid publication on acceptance

- support for research data, including large and complex data types

- gold Open Access which fosters wider collaboration and increased citations

- maximum visibility for your research: over $100 \mathrm{M}$ website views per year

At BMC, research is always in progress.

Learn more biomedcentral.com/submissions 